\title{
EchoGéo
}

15 | 2011

La Belgique, État prospère en décomposition au cœur de l'Europe du nord-ouest

\section{Caractérisation interrégionale des paysages de la Belgique}

\section{Veerle Van Eetvelde et Marc Antrop}

\section{(2) OpenEdition}

\section{Journals}

Édition électronique

URL : https://journals.openedition.org/echogeo/12266

DOI : $10.4000 /$ echogeo.12266

ISSN : 1963-1197

Éditeur

Pôle de recherche pour l'organisation et la diffusion de l'information géographique (CNRS UMR 8586)

Référence électronique

Veerle Van Eetvelde et Marc Antrop, « Caractérisation interrégionale des paysages de la Belgique », EchoGéo [En ligne], 15 | 2011, mis en ligne le 11 avril 2011, consulté le 03 août 2021. URL : http:// journals.openedition.org/echogeo/12266 ; DOI : https://doi.org/10.4000/echogeo.12266

Ce document a été généré automatiquement le 3 août 2021.

EchoGéo est mis à disposition selon les termes de la licence Creative Commons Attribution - Pas d'Utilisation Commerciale - Pas de Modification 4.0 International (CC BY-NC-ND) 


\title{
Caractérisation interrégionale des paysages de la Belgique
}

\author{
Veerle Van Eetvelde et Marc Antrop
}

Les auteurs remercient Mme Dominique Godfroid pour les corrections linguistiques.

\section{Introduction}

La diversité des paysages en Belgique est exceptionnellement grande et est le résultat d'une grande variation physique et naturelle et d'une longue histoire multiculturelle mouvementée. Avec cinq phases successives de fédéralisation depuis 1970, un aperçu général de la Belgique dans un contexte européen est devenu graduellement de plus en plus rare. Ceci est certainement le cas pour des aspects dont la politique a été régionalisée depuis 1980 comme l'aménagement du territoire, l'environnement, le patrimoine, dont le paysage. Les grandes unités paysagères sont axées sur la structure physique du pays et ont été coupées transversalement par les divisions politiques et administratives. Par conséquent, des unités paysagères homogènes ont été coupées en deux et connaissent une gestion différente depuis déjà 30 ans. En outre, le développement économique et régional, l'impact de l'urbanisation et les infrastructures de transport sur la transformation du milieu rural diffèrent dans les trois régions. Cet article présente une caractérisation actuelle et interrégionale des paysages de la Belgique entière.

\section{La structure paysagère naturelle et historique}

La Belgique s'étend de la Mer du Nord à l'Ardenne, massif hercynien ancien. La géologie varie d'une façon très complète du Primaire au Quaternaire. Le pays a été façonné par toutes les orogenèses importantes, transgressé par les mers et couvert de sédiments divers et finalement il fut remodelé dans les périodes périglaciaires. Une nature variée résulte de cette diversité de la lithologie, de la géomorphologie et des sols. La structure générale est un gradient orienté du sud-est au nord-ouest, des hauts plateaux 
ardennais aux polders et vers la plaine côtière. Le développement du réseau hydrographique a suivi ce gradient en s'adaptant à la condition lithologique (Maréchal, 1992 ; De Moor et Pissart, 1992). Six grandes unités orographiques se distinguent par leur altitude moyenne (figure 1). A l'ouest et au nord, la plaine côtière et les polders sont limités par l'isohypse de 5 mètres (zone I). Plus à l'intérieur (zone II), une plaine s'élève jusqu'à 50 mètres où dominent les sols sablonneux de la Flandre et de la Campine et est bordée par la terrasse fluviale de la Campine à l'est. Au centre, des basplateaux sablo-limoneux et limoneux du Pays des Collines, du Brabant et de l'Hesbaye s'élèvent jusqu'à environ 200 mètres (zone III). Plus au sud s'étendent les plateaux du Hainaut, de l'Entre-Sambre-et-Meuse, du Condroz, ainsi que la dépression de la FagneFamenne et la Calestienne (zone IV). Ici des sols limons-caillouteux dominent. Au sud de la région calcaire de la Calestienne, le terrain s'élève vers le haut plateau des Ardennes qui s'étend de 400 à 694 mètres (zone V). A l'extrémité sud du pays commencent les cuestas du Luxembourg (zone VI).

Figure 1 - Carte du relief avec hydrographie et les frontières administratives des Régions (Flandre, Wallonie, Bruxelles-Capitale)

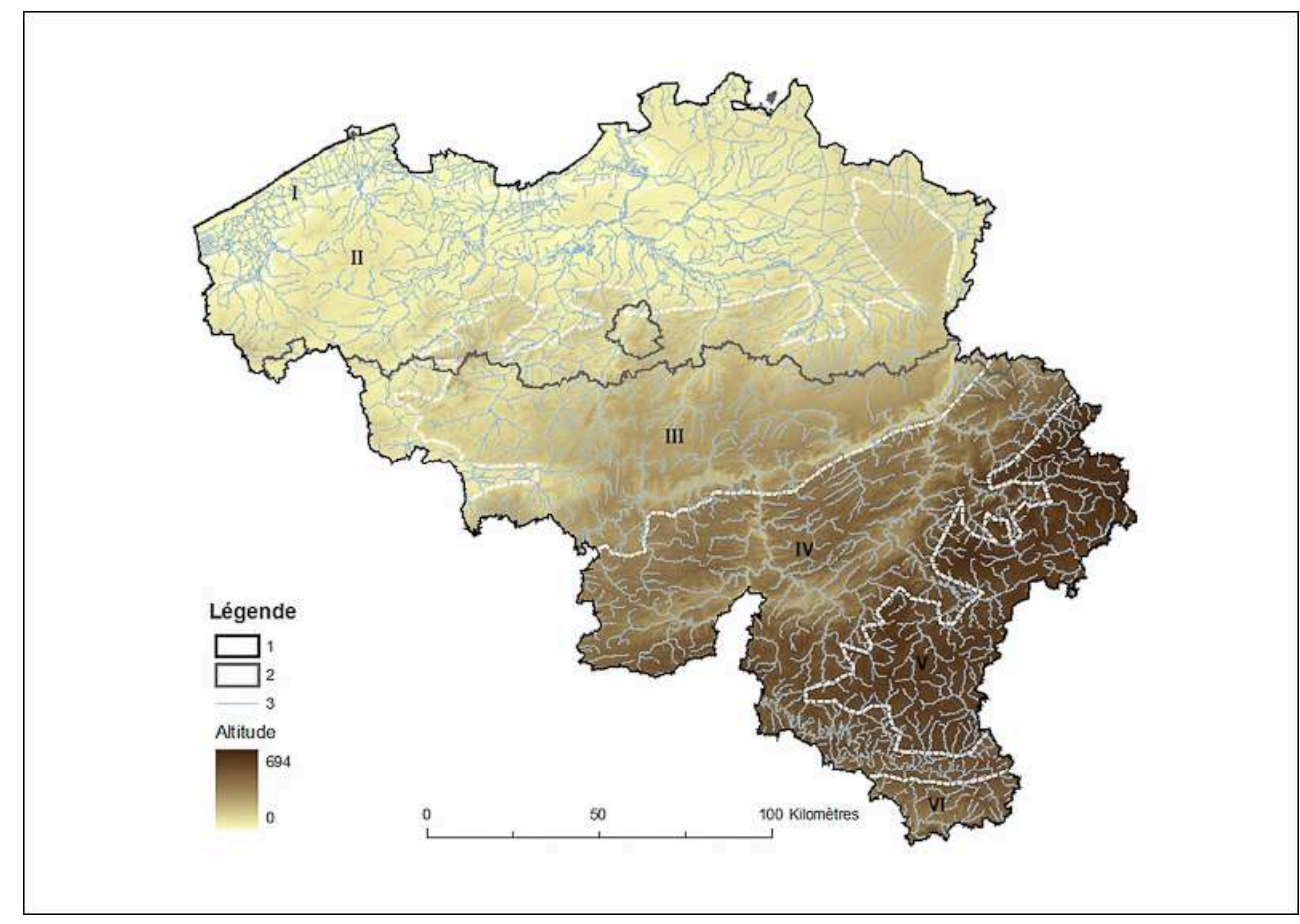

1 : la frontière de la Belgique ; 2 : les frontières des Régions ; 3 : I'hydrographie ; 4 : l'altitude. Les caractères romains se réfèrent à six grandes unités orographiques.

La plaine côtière, qui s'étend du Pas-de-Calais vers les pays baltiques, et les plateaux limoneux fertiles formaient durant toute l'histoire un couloir nord-sud et ouest-est. Le territoire était ainsi une zone d'échanges culturels et de confrontations avec des civilisations différentes et souvent violentes. Les emprunts à ces cultures diverses ont augmenté la diversité naturelle. Ainsi des régions et paysages aux caractères distincts ayant une étonnante diversité se sont formés dans un espace géographique très restreint (Christians et Daels, 1988 ; Antrop et al., 2006). La transition entre une culture romane et germanique est un facteur sans doute important qui s'est développé aujourd'hui en une frontière linguistique et politique dure. 
Le concept des paysages traditionnels évoque cette diversité paysagère qui s'est développée graduellement pendant deux millénaires jusqu'aux grands changements dévastateurs qui ont débuté il y a environ 300 ans suite aux importantes réformes agraires, à la révolution industrielle et aux changements sociaux et politiques qu'elle engendrait (Antrop, 1997). Les paysages traditionnels sont caractérisés par le résultat de l'interaction entre les qualités naturelles (relief, sols, géologie,...) et l'occupation par l'homme (habitat, utilisation du sol,...) à travers l'histoire et avant les nombreux changements modernes, du début du XIX ${ }^{e}$ siècle (Antrop, 1997). L'état et l'évolution des paysages sont bien représentés dans les premières cartes topographiques par la carte de cabinet du comte de Ferraris (fin du XVIII ${ }^{e}$ siècle) qui est une référence importante. C'est depuis la seconde moitié du XIX ${ }^{e}$, que les changements se sont surtout accélérés et ont profondément affecté, et même rasé, les paysages. Aujourd'hui, les structures paysagères anciennes n'existent quasi plus que par des fragments isolés, dépourvus d'un contexte géographique et structurel et par conséquent de moins en moins fonctionnels et perdant leur identité. Ainsi, les paysages traditionnels évoquent les qualités et les valeurs des paysages comme patrimoine naturel et culturel important. Le concept des paysages traditionnels a été utilisé en Région flamande d'une part pour reconstituer ce cadre spatial géographique et historique, et d'autre part pour formuler des modèles systémiques de différents types de paysages décrivant les potentialités naturelles des différents environnements. Ce cadre spatial n'est rien d'autre qu'une fine classification chorologique des territoires historiques et des régions géographiques classiques (figure 2). La délimitation des unités se fait ici par des limites plus perceptibles et reconnaissables sur le terrain. 
Figure 2 - Typologie paysagère de la région Flamande, Wallonne et Bruxelles-Capitale

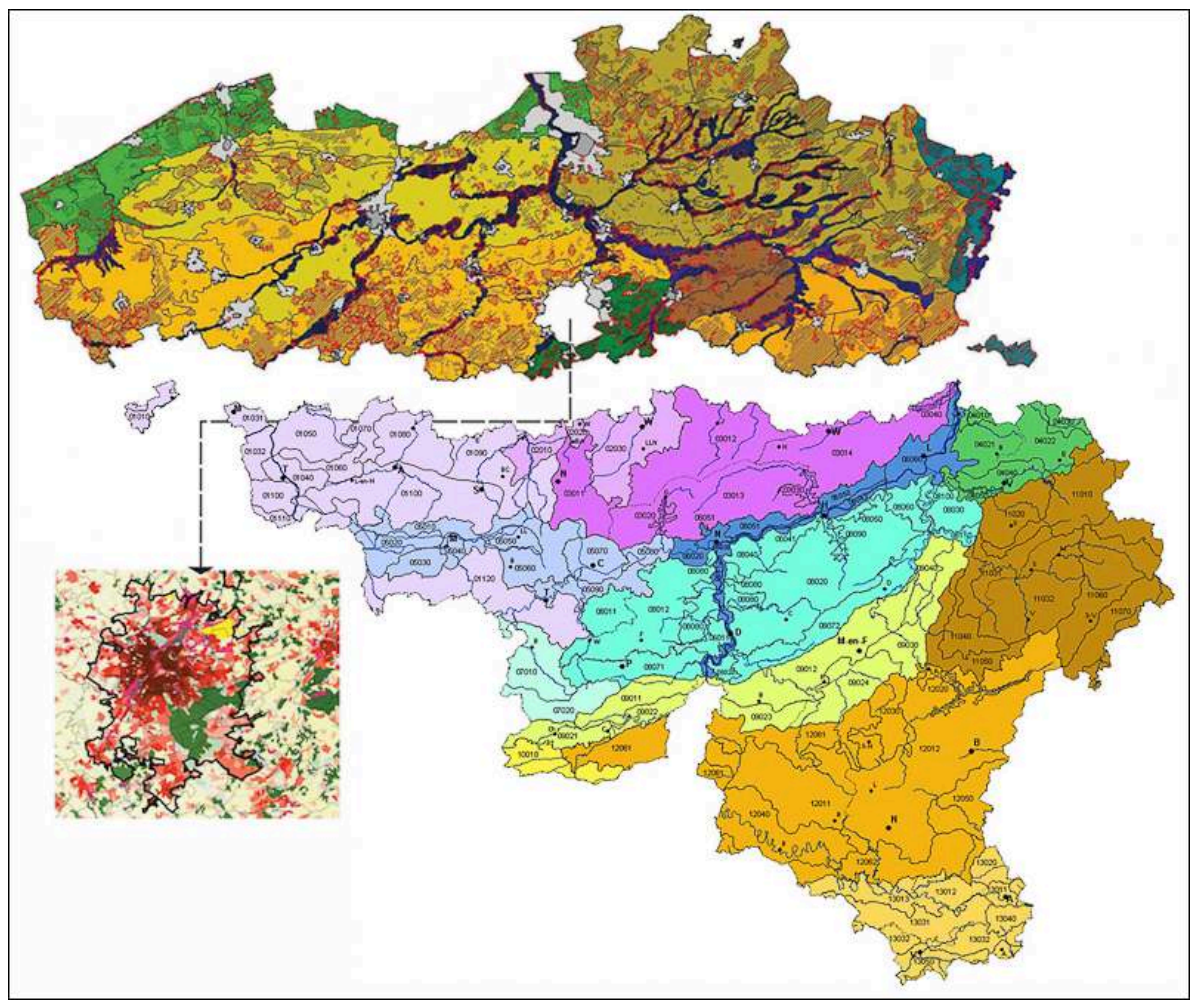

Illustration du haut : les 124 paysages traditionnels de la Flandre (Antrop et al., 2002). 23 régions paysagères sont représentées en couleur ; les zones de reliquats de l'Atlas paysager flamand (AGIV, 2004) sont marquées par des traits noirs et les places d'ancrages sont bordées de rouge. Illustration du bas : les 79 territoires paysagers de la région wallonne (Droeven et al., 2004). 13 ensembles paysagers sont représentés en couleur.

\section{Les grandes transformations}

Bien que des grandes innovations dans l'agriculture aient façonné le paysage depuis le bas-Moyen-Age, les changements les plus importants, sont survenus fin du XVIII ${ }^{\mathrm{e}}$ siècle. Des révolutions technologiques, sociales et politiques causent des transformations de l'industrie et du mode de vie. En Flandre l'industrie textile et en Wallonie l'exploitation minière et l'industrie du fer furent modernisées. La campagne devient le terrain de recrutement pour la main-d'œuvre et la zone de culture pour l'alimentation des centres urbains et industriels en expansion. Le jeune état belge, voulant s'affirmer comme nation moderne, se dote d'un réseau dense de canaux et chemins de fer, permettant une mobilité croissante pour toute la population (Antrop et al., 2010). Aussi, l'État oblige, sous peine d'amende, les propriétaires et les communes, à défricher les terrains sauvages et incultes afin de nourrir la population croissante et d'augmenter la production de bois. Ainsi, beaucoup d'aires de pâture et de landes, comme les wastines médiévales sont reboisées avec des pins. L'importation massive de blé moins coûteuse à l'étranger (invasion agricole) par les propriétaires d'usines en compétition économique rude, oblige les agriculteurs à modifier l'utilisation du sol. Ils se spécialisent en légumes et fruits, et dans l'élevage. Localement des vastes surfaces de terrains arables sont transformées en prairies dans un paysage bocager. L'exode rural cause une densification des villes historiques industrialisées formant des ceintures ou 
conurbations industrielles. Les réseaux denses des chemins de fer et surtout des chemins de fer vicinaux, dont la longueur atteint 58 kilomètres en 1885 jusqu'à 4756 kilomètres en 1939, permettent le désenclavement de communes rurales même situées dans la périphérie. Ainsi, ils initient une première phase de péri-urbanisation. Après la Deuxième Guerre mondiale, c'est l'automobile qui prend la relève causant une périurbanisation rapide autours des grands centres urbains et leurs routes d'accès. Tandis que les premières zones industrielles ainsi que les régions minières étaient concentrées dans les villes industrialisées et portuaires, l'industrie d'après guerre se localise dans les zones portuaires et le long des nouvelles autoroutes et aéroports. La figure 3 montre l'importance de la péri-urbanisation et l'effet de la fragmentation de l'espace rural. Le contraste entre le nord et le sud du pays est net. Au nord, en Flandre en particulier, la péri-urbanisation domine le paysage actuel qui est caractérisé par une forte densité de villes historiques et de routes.

Figure 3 - Évolution de l'urbanisation

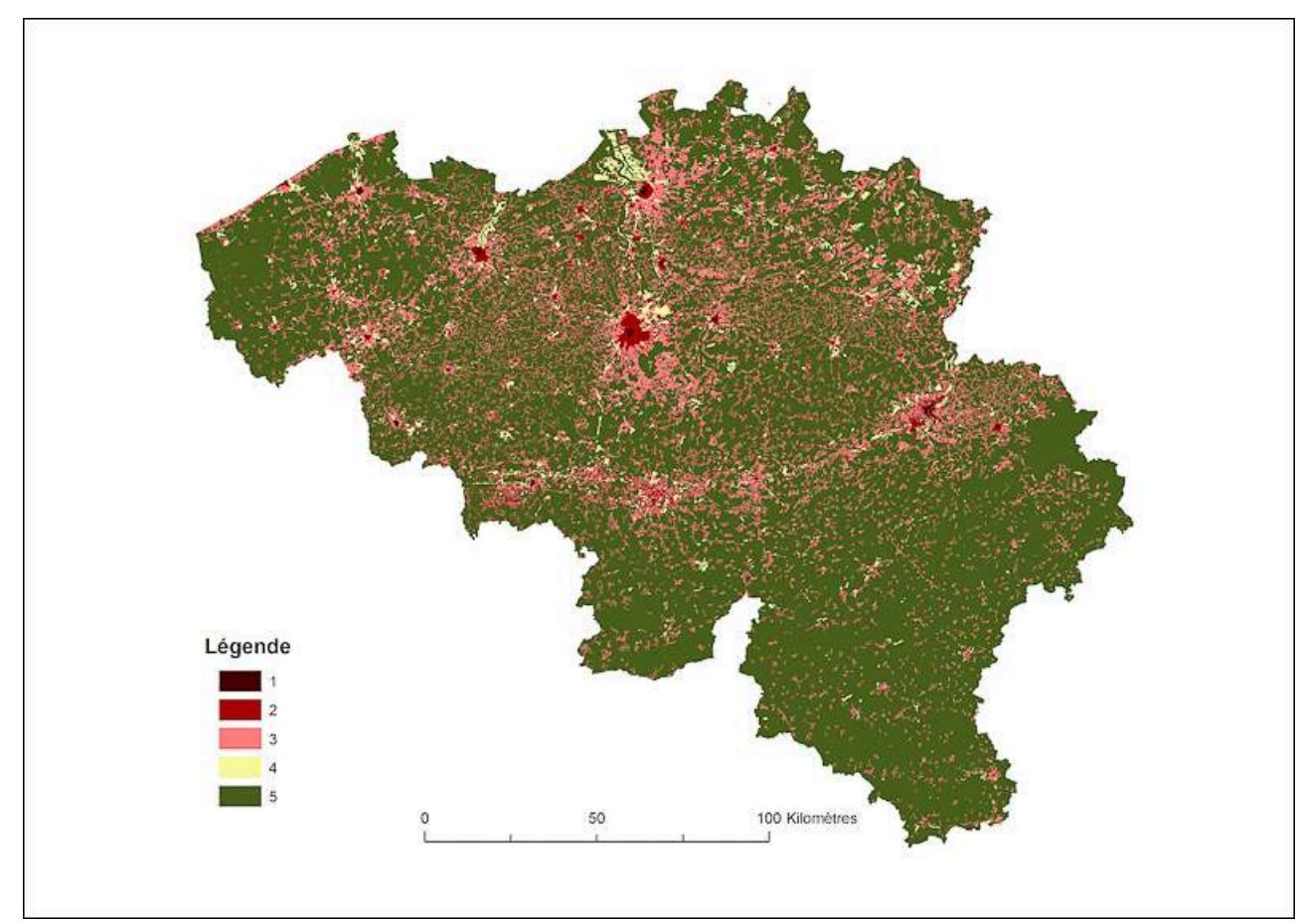

1 : centres historiques $X V I I I$; 2 : extension urbaine $X I X$; 3 : urbanisation et péri-urbanisation $X X^{e}-X X I^{e}$; $4:$ zones industrielles actuelles ; 5 : les paysages ruraux et forestiers.

\section{Des nouvelles frontières politiques et différentes approches du paysage}

En Belgique, les communautés ont obtenu leur autonomie culturelle en 1970 et les régions sont devenues autonomes en 1980 en ce qui concerne le territoire (Marissal et al., 2007). Depuis, la frontière linguistique est devenue une frontière politique, partageant en deux les bassins hydrographiques et les paysages traditionnels. Les politiques, l'aménagement du territoire et la gestion du patrimoine et des paysages ont divergé graduellement entre le nord et le sud du pays. Quant au paysage, différentes approches et concepts se sont développés. Ceux-ci sont par exemple bien 
reconnaissables dans la mise en oeuvre de la convention européenne du paysage (Council of Europe, 2000), en ce qui concerne l'identification des paysages en particulier. Les typologies différentes développées dans la région flamande et wallonne sont comparées dans la figure 2 (Antrop et al., 2004).

En Flandre, une description et une cartographie des paysages traditionnels existaient déjà et avaient été utilisées pour l'élaboration du nouveau plan structurel d'aménagement du territoire en 1993 (Antrop, 1996). C'était une typologie et chorologie holistique et hiérarchique se basant sur des cartes topographiques historiques et des photographies aériennes avec une description générale des unités paysagères (figure 2 haut). Cette classification formait la base pour l'élaboration de l'Atlas paysager flamand, appelé plus précisément "Atlas des reliquats des paysages traditionnels en Flandre". Le but était d'établir un inventaire des caractéristiques historiques et culturels encore existantes, semblable à l'inventaire biologique, déjà existant, du milieu naturel .Méthodologiquement, l'approche était holistique et sélective, cartographiée à l'échelle 1/50 000 (Antrop et Van Eetvelde, 2007). Techniquement, l'atlas est une base de données SIG disponibles en CD-ROM et par le géo-portail de l'Agence Information Géographique Flamand (AGIV, 2004). La première version de cet atlas a été publiée en 2001 par le ministère flamand, et dans l'élan de la Convention européenne du paysage, un premier pas vers une gestion paysagère intégrale a été lancé (Hofkens et Roossens, 2001). Depuis 2004, l'Institut Flamand pour l'Héritage Immobilier (VIOE) est chargé de l'actualisation et de la gestion de l'Atlas.

En Wallonie, la conception du paysage s'orientait plutôt vers un environnement perçu et un cadre de vie actuel et dynamique. L'aspect tangible (visuel) et le patrimoine bâti (architecture) sensoriel sont importants. C'est la Conférence Permanente du Développement Territorial (CPDT) qui a pris en charge la direction et la coordination du travail. En 2004, la classification des "territoires paysagers de la Wallonie" fut réalisée (Droeven et al., 2004) au moyen d'une cartographie à échelle 1/250 000 et d'une classification hiérarchisée des territoires formant des groupes d'ensembles paysagers (figure 2 bas). En 2008, parut le premier tome des Atlas des paysages de Wallonie sous forme de monographies bien illustrées, pour les ensembles paysagers de l'EntreVesdre-et-Meuse (Cremasco et al., 2007) et des Plateaux Brabançon et Hesbignon (De Witte et al., 2009).

Il faut remarquer également que la région Bruxelles-Capitale est une unité territoriale autonome dans une Belgique fédérale, caractérisée surtout par des paysages urbains. Une typologie paysagère officielle pour la région Bruxelles-Capitale n'existant pas, on a inséré dans la figure 2 une classification des secteurs statistiques basée sur l'utilisation dominante du sol et combinée avec la densité des populations. C'est ainsi que les cartes de la Région Flamande, ont un "trou blanc" à l'endroit où la Région Bruxelles-Capitale est située.

\section{Les paysages actuels de la Belgique}

Le caractère des paysages actuels de la Belgique est essentiellement déterminé par des contrastes perceptibles : bâti/non bâti, industrie/sans industrie, urbain/rural, plat/ onduleux, homogène/hétérogène, grande/petite échelle, eau/terre, ouvert/fermé. Une typologie doit spécifier ces contrastes. La nouvelle typologie des paysages couvrant toute la Belgique à été élaborée afin de l'insérer dans la typologie européenne (Mücher 
et al., 2003, 2006), conformément à la Convention européenne du paysage (Council of Europe, 2000). Cette typologie au niveau fédéral est basée sur la méthodologie de la caractérisation des paysages initialement développée en Angleterre et en Écosse (Landscape Character Assessment (LCA) Swanwick, 2004). La caractérisation se fait sur deux échelles et en deux phases. Des types de paysages sont définis et sont ensuite groupés en aires paysagères. Dans le cas de la Belgique, la première échelle consiste en une typification de cellules d'un kilomètre carré. La composition et la configuration des cellules forment la base pour la délinéation des unités paysagères à la deuxième échelle (Van Eetvelde et Antrop, 2009). La figure 4 illustre le schéma méthodologique et la figure 5 le résultat.

Figure 4 - Méthodologie pour la caractérisation des paysages à deux échelles

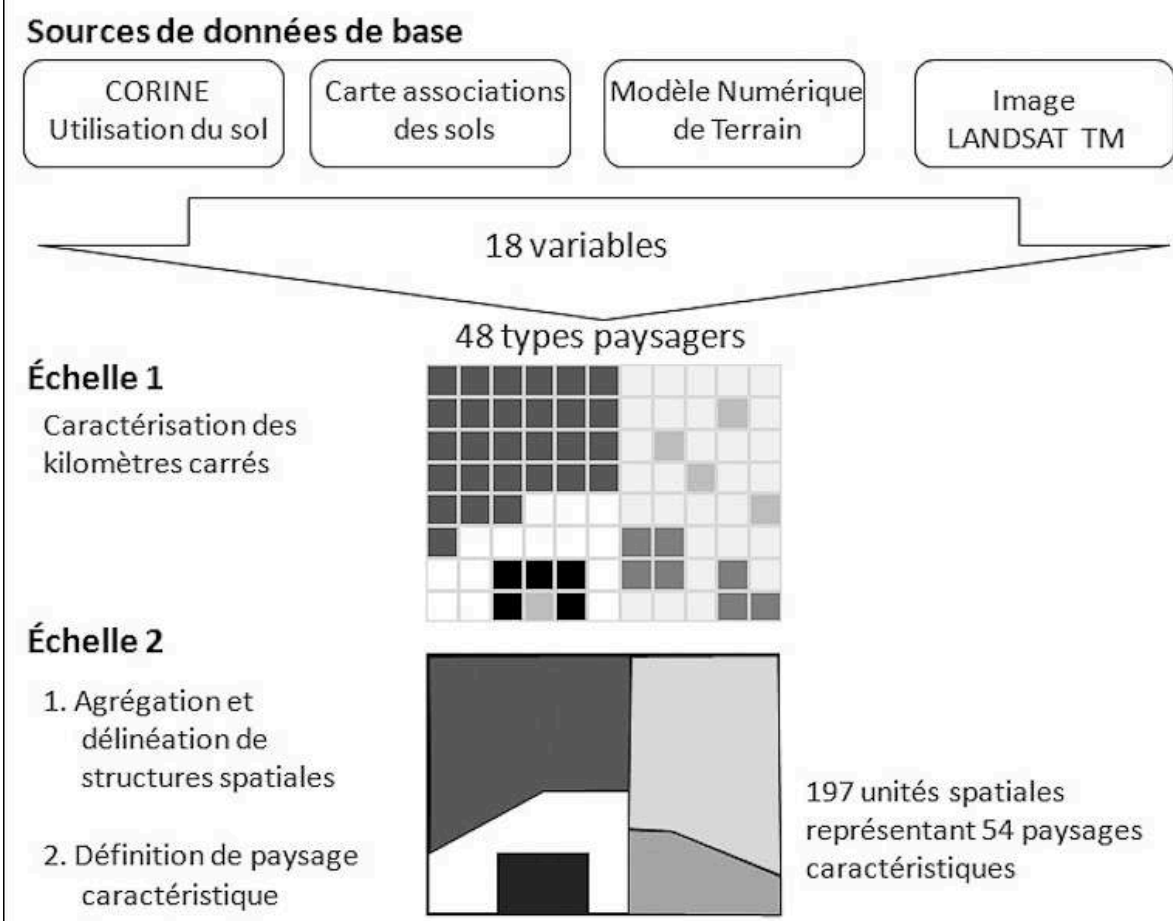

Seulement quatre sources de données numériques ayant un détail d'information suffisant sont disponibles et couvrent toute la Belgique. Il s'agit de l'utilisation du sol, de la base de données CORINE Land Cover de 1990, de la carte d'association des sols, du modèle numérique de terrain, et des images satellitaires, telle que l'image composite du Landsat TM de 1989-1990 qui a été utilisée. Les données CORINE Land Cover permettent de distinguer les zones bâties et non bâties, les espaces ouverts, ainsi que les parties industrielles, les cultures, les pâturages, les forêts et les surfaces aquatiques. La carte d'association des sols fournit l'information concernant le substrat physique, ce qui est déterminant pour l'utilisation $\mathrm{du}$ sol. Le modèle d'altitude numérique permet de déduire la variation d'altitude ainsi que l'altitude moyenne, ceci en vue de pouvoir distinguer les zones planes qui sont situées ou non en hauteur. L'image satellite ne contient aucune donnée thématique et son échelle est petite, mais elle contient suffisamment de détails (résolution $25 \mathrm{~m}$ ) pour pouvoir estimer l'hétérogénéité paysagère, ainsi que les principaux modèles et formes de parcelles. Par kilomètre carré, 
on détermine l'entropie de l'image qui exprime l'hétérogénéité de la diversité paysagère, les paysages très hétérogènes fournissent des valeurs d'entropie élevées.

Chaque kilomètre carré est, à la première échelle, représenté par 18 variables, dérivées de quatre sources de données. Pour l'intégration spatiale de l'occupation du sol (6 variables) et des associations des sols (9 variables), la proportion de superficie occupée par chaque catégorie a été calculée par kilomètre carré. Le relief et la topographie sont représentés par l'altitude moyenne et par la variation d'altitude dans une cellule d'un kilomètre carré. Enfin, l'hétérogénéité de la diversité paysagère est dérivée de l'image satellite. En utilisant les 18 variables il est possible de définir 48 types de paysages distincts. Pour la présentation cartographique, chaque type reçoit une couleur déterminée par la variable la plus caractéristique (figure 5a). Ainsi, les types possédant plus de $40 \%$ de superficie bâtie reçoivent par exemple une gradation du rouge au rose.

Pour la deuxième échelle, les 31433 kilomètres carrés de la Belgique caractérisés à la première échelle sont ensuite agrégés d'une façon holistique pour former 197 unités spatiales qui consistent en une composition et configuration de cellules caractéristiques d'un kilomètre carré. Ainsi, 54 paysages à caractère typique peuvent être définis et sont illustrés dans la figure $5 b$. Plusieurs unités spatiales peuvent donc posséder le même caractère paysager. Les paysages urbains sont composés des types 1 à 3 représentant les agglomérations urbaines et les villes à extension péri-urbaine importante. Elles apparaissent en 29 aires. Les paysages péri-urbanisés sont caractérisés par les types 4-7, qui contiennent un mixage de bâti et d'espaces ruraux occupant 29 aires. Cinq aires de paysages industrialisés et portuaires groupent les types 8 et 9 . La côte forme un type à part $\left(n^{\circ} 10\right)$ et est fragmentée en trois unités. Les polders consistent en deux types paysagers : les polders de la plaine maritime (type 11, A sur figure 5a) et les polders fluviaux le long de l'Escaut (type 12), fragmentés en cinq unités spatiales. La majeure partie de la Belgique centrale est occupée par des paysages agraires à cultures prédominantes, formant 74 aires paysagères. Elles sont caractérisées par différentes combinaisons de 18 types paysagers (types 13 à 30) de cultures, prairies, forêts et terrains bâtis. Les paysages dominés par les herbages (7 types, 31-36) se situent en Campine (D), en Fagne (I), dans la partie orientale des Ardennes (L) et en Lorraine $(\mathrm{N})$. Les paysages forestiers sont formés de 14 types paysagers (37-50) et comprennent des paysages dominés par la forêt ainsi que des mélanges de forêt, de landes, de pâturages, de cultures et du bâti. Elles forment 35 aires paysagères caractéristiques situées en Campine (D), en Fagne et en Famenne (I et J), en Ardennes (L) et dans les cuestas de la Lorraine (N). Enfin, il y a les paysages des vallées, formés de quatre types (51-54), caractérisés par une utilisation du sol hétérogène et fragmenté.

A une échelle plus détaillée, la caractérisation des kilomètres carrés, représentation cartographique en grille (figure 5a), permet aussi d'identifier quelques structures typiques. Par exemple, les Polders (A) se caractérisent par deux types de paysages seulement (un à dominance de cultures et l'autre à dominance de prairies) et forment l'aire paysagère $n^{\circ} 11$ sur la carte. Les paysages bocagers de la région sablonneuse de la Flandre septentrionale (B) se distinguent structurellement des paysages bocagers des plateaux limoneux (C), formant deux aires à caractéristique différente (aires 13 et 16). En Campine au nord (D) l'aire paysagère $n^{\circ} 15$, se caractérise par une mosaïque grossière de champs ouverts, bois et zones urbanisées formant un paysage hétérogène. Les paysages sur les plateaux limoneux (aires 19 et 20) consistent en des openfields qui sont plus homogènes dans la partie orientale $(E)$ que dans la partie occidentale $(F)$ où ils sont plus morcelés. Le paysage (aire 25) du plateau ondulé du Condroz (G) à grandes 
fermes se distingue nettement du sillon industriel de la Sambre et Meuse ( $\mathrm{H}$, aire caractéristique 26) au nord, ainsi que des collines calcaires et boisées de la Calestienne (K) au sud. Les Ardennes se composent de longues pentes raides et disséquées ( $\mathrm{M}$, aire paysagère 43) et de hauts plateaux (L) caractérisés par un mélange de prairies et forêts donnant l'aire paysagère 27.

Figure 5 - Typologie paysagère de la Belgique à deux échelles

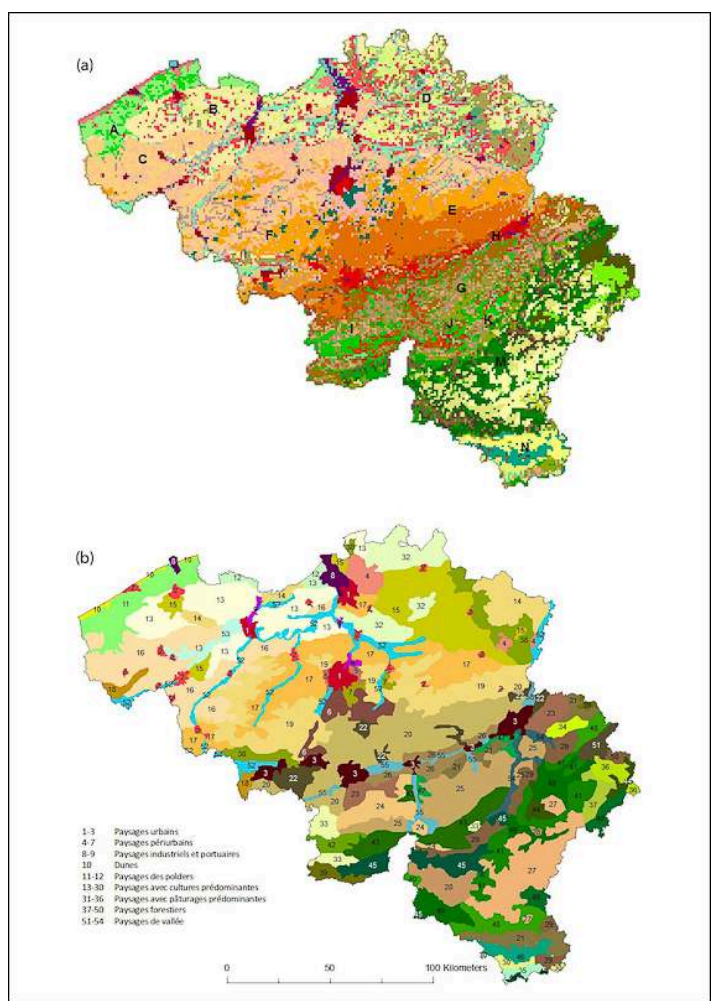

(a) Typologie paysagère des cellules de $1 \mathrm{~km}^{2}$. Les lettres indiquent les régions géographiques, référées dans le texte. (b) Unités paysagères.

\section{Conclusion}

La diversité naturelle et culturelle des paysages traditionnels de la Belgique a été fragmentée par les transformations importantes des trois siècles derniers. De nouveaux paysages se sont formés, remplaçant parfois les paysages existants, plus particulièrement en transformant leur caractère. Cette évolution était plus importante dans les régions fortement peuplées et urbanisées. Ainsi le contraste nord-sud s'est accentué en créant dans les trois régions de l'état fédéral des situations très différentes pour l'aménagement et la gestion des paysages. 


\section{BIBLIOGRAPHIE}

AGIV, 2004. http://www.geo-vlaanderen.gisvlaanderen.be/geo-vlaanderen/landschapsatlas/

Antrop M., 1996. Des régions géographiques aux paysages traditionnels et paysages culturels futurs. Société Géographique de Liège, No. Hors série, J.-P.Donnay et C.Chevigné (éditeurs), Recherches de géographie humaine. Hommage au Professeur Ch. Christians, p. 261-268

Antrop M., 1997. The concept of traditional landscapes as a base for landscape evaluation and planning. The example of Flanders Region. Landscape and Urban Planning, $\mathrm{n}^{\circ} 38$, p. 105-117.

Antrop M., De Maeyer Ph., Vandermotten Ch., Beyaert M., Billen C., Decroly J.-M., Neuray C., Ongena T., Queriat S., Van Den Steen I., Wayens B., 2006. België in kaart. De evolutie van het landschap in drie eeuwen cartografie. NGI-Lannoo, Brussel/Tielt, $250 \mathrm{p}$.

Antrop M., Van Eetvelde V., Janssens J., Martens I., Van Damme S., 2002. Traditionele Landschappen van Vlaanderen. Universiteit Gent, Vakgroep Geografie. http://www.geoweb.ugent.be/ landschapskunde/projecten/traditionele-landschappen-vlaanderen

Antrop M., Belayew D., Droeven E., Felts C., Kummert M., Van Eetvelde V., 2004. Landscape research in Belgium. Belgeo, $\mathrm{n}^{\circ}$ 2-3, p. 205-218.

Antrop M., Van Eetvelde V, 2007. L'atlas des paysages flamands dans la politique intégrée d'aménagement du territoire. In Berlan-Darqué M., Luginbühl Y., Terrason D. (Eds.) Paysages : de la connaissance à l'action. Editions Quae, p. 139-148.

Antrop M., Schmitz S., Sevenant M., Van Eetvelde V., Van Hecke E., 2010. Atlas de Belgique - Tome 2 Paysages, monde rural et agriculture. Academia Press, 74 p.

Christians C., Daels L., 1988. Belgium: an introduction to its regional diversity and cultural richness. Société Géographique de Liège, $n^{\circ} 24,80$.

Council of Europe, 2000. European Landscape Convention and Explanatory Report. Council of Europe, Document by the Secretary General established by the General Directorate of Education, Culture, Sport and Youth, and Environment, Florence.

Cremasco V., Doguet A., Feremans N., Neuray C., Pons T., Van der Kaa C., Godart, M.F., Feltz C., 2007. Atlas des Paysages de Wallonie, 1. L'Entre-Vesdre-et-Meuse. La Conférence Permanente du Développement Territorial, Ministère de la Région wallonne.

De Moor G., Pissart A., 1992. Het reliëf. In Denis, J., (Ed.), Geografie van België. Gemeentekrediet, Brussel, p. 129-215.

De Witte C., Neuray C., Nielsen M., Pons T., Van der Kaa C., Godart M.F., Feltz C., 2009.Atlas des Paysages de Wallonie, 2. Les Plateaux brabançon et hesbignon. La Conférence Permanente du Développement Territorial, Ministère de la Région wallonne.

Droeven E., Feltz C., Kummert M., 2004. Les territoires paysagers de Wallonie. Namur, Etudes et Documents, CPDT, La Conférence Permanente du Développement Territorial, 4, 68 p.

Hofkens, E. \& Roossens, I. (eds.), 2001. Nieuwe impulsen voor de landschapszorg. De landschapsatlas, baken voor een verruimd beleid. Ministerie van de Vlaamse Gemeenschap, Afdeling Monumenten en Landschappen, Brussel, $191 \mathrm{p}$.

Marissal P., Lockhart P.M., Van Hamme G., Vandermotten C., 2007. Atlas de Belgique. 1-Géographie Politique. Gent, Academia Press, 32 p. 
Maréchal R., 1992. De geologische structuur. In Denis J. (Ed.) Geografie van België. Brussel, Gemeentekrediet, p. 38-86.

Mücher C. A., Bunce R. G. H., Jongman R. H. G., Klijn J. A., Koomen A. J. M., Metzger M. J., Washer D. M. 2003. Identification and Characterisation of Environments and Landscapes in Europe. Alterra, rapport 832, Wageningen.

Mücher C. A., Wascher D. M., Klijn J. A., Koomen A. J. M. Jongman, R. H. G., 2006. A new European Landscape Map as in integrative framework for landscape character assessment. In Bunce R. G. H., Jongman R. H. G. (Eds.), Landscape Ecology in the Mediterranean: inside and outside approaches. Proceedings of the European IALE Conference, 29 March - 2 April 2005, Faro, Portugal. IALE Publication Series 3, pp. 233-243.

Swanwick C., 2004. Landscape Character Assessment. Guidance for England and Scotland. Topic Paper 6 Techniques and criteria for judging capacity and sensitivity. The Countryside Agency, Scottish Natural Heritage.

Van Eetvelde V., Antrop M., 2005. Landscape Character Assessment in Belgium: Balancing Natural and Cultural Properties.De Belgische Geografendagen, Deel II, BEVAS-SOBEG, Gent, p. 347-353.

Van Eetvelde V., Antrop M., 2009. A stepwise multi-scaled landscape typology and characterisation for trans-regional integration, applied on the federal state of Belgium.Landscape and Urban Planning, 91, p. $160-170$.

VIOE. http://www.vioe.be/

\section{RÉSUMÉS}

La diversité des paysages en Belgique est exceptionnellement grande et est le résultat d'une grande variation physique et naturelle et d'une longue histoire multiculturelle mouvementée. Avec la fédéralisation successive de la Belgique, il devient de plus en plus difficile d'obtenir des aperçus de synthèse du pays, surtout en ce qui concerne les matières régionalisées comme l'aménagement $d u$ territoire et la gestion du patrimoine en particulier. Depuis 1980, les politiques concernant les paysages sont divergentes et ont résulté en des conceptions et approches différentes. Ceci se manifeste clairement dans la mise en œuvre de la Convention européenne du paysage, en particulier dans l'identification et la gestion des paysages. Une synthèse interrégionale sur la typologie des paysages actuels a été réalisée en utilisant les sources de données communes.

The landscapes in Belgium are exceptional diverse due to the important variation of the physical and natural conditions as well as to the long multicultural and tormented history. Belgium became gradually a federal state which made it difficult to get overall views on matters that became by regional governance such as spatial planning and heritage and landscape management. Since 1980 policies are diverging between the different regions and different concepts and approaches are used for landscape management and planning. This becomes obvious looking at the implementation of the European Landscape Convention in the three regions, in particular with the identification of landscapes. Although data sources covering the whole of Belgium have become rare, an interregional synthesis and landscape characterization for the whole of the country is presented. 


\section{INDEX}

Mots-clés: Bruxelles-Capitale, convention européenne du paysage, Flandres, identification paysages, Wallonie

Keywords : Brussels Capital City, European Landscape Convention, Flanders, landscape identification, Wallonia

\section{AUTEURS}

\section{VEERLE VAN EETVELDE}

Veerle Van Eetvelde est Professeur à l'Université de Gand, Département de Géographie. veerle.vaneetvelde@ugent.be

\section{MARC ANTROP}

Marc Antrop est Professeur émérite à l'Université de Gand, Département de Géographie. marc.antrop@ugent.be

Ils ont publié récemment :

- Antrop M., Belayew D., Droeven E., Felts C., Kummert M., Van Eetvelde V., 2004. Landscape research in Belgium. Belgeo, $\mathrm{n}^{\circ}$ 2-3, p. 205-218.

- Van Eetvelde V., Antrop M. 2009. A stepwise multi-scaled landscape typology and characterisation for trans-regional integration, applied on the federal state of Belgium. Landscape and Urban Planning, ${ }^{\circ}$ 91, p. 160-170

- Van Eetvelde V., Antrop M., 2009. Indicators for assessing changing landscape character of cultural landscapes in Flanders (Belgium). Land Use Policy, n² 27, p. 901-910.

- Van Hecke E., Antrop M., Schmitz S., Van Eetvelde V., Sevenant M., 2010. Atlas van België. Deel 2 : Landschap, platteland en landbouw, Academia Press. 